Check for updates

Cite this: RSC Adv., 2017, 7, 32461

\title{
Introducing $\mathrm{Ti}^{3+}$ defects based on lattice distortion for enhanced visible light photoreactivity in $\mathrm{TiO}_{2}$ microspheres $\dagger$
}

\begin{abstract}
Yunfan Xu, (D) ab Sujuan Wu, ${ }^{* a}$ Piaopiao Wan, ${ }^{a}$ Jianguo Sun ${ }^{\text {a }}$ and Zachary D. Hood ${ }^{\text {cd }}$
Defective titanium dioxide $\left(\mathrm{TiO}_{2}\right)$ is of much significance due to its improved visible light photoreactivity. Generally, the existence of defects leads to imperfections in the crystal lattice, which in turn affect the dynamics of the evolution of defects and the corresponding physical properties of $\mathrm{TiO}_{2}$. Until now, how lattice distortion affects the formation of $\mathrm{Ti}^{3+}$ defects as well as the corresponding visible light photoreactivity in $\mathrm{TiO}_{2}$ has remained elusive. Herein, we have successfully introduced $\mathrm{Ti}^{3+}$ defects based on lattice distortion in $\mathrm{TiO}_{2}$ microspheres and found the photocurrent of anatase $\mathrm{TiO}_{2}$ has been significantly enhanced from 1.78 to $80 \mu \mathrm{A} \mathrm{cm}{ }^{-2}$ with an increase in photocatalytic activity of almost three times under visible light irradiation. Furthermore, we show that lattice distortions have minimal contribution to enhancing the visible light photocatalytic activity because the band gap cannot be narrowed due to the absence of $\mathrm{Ti}^{3+}$ defects, yet the existence of lattice distortions could suppress the recombination of electron-hole pairs. Moreover, the formation of $\mathrm{Ti}^{3+}$ defects is energetically favored in lattice-distorted $\mathrm{TiO}_{2}$ compared to that of pristine $\mathrm{TiO}_{2}$. This work highlights the design and development of highly efficient $\mathrm{TiO}_{2}$ photocatalysts that operate under visible light irradiation.
\end{abstract}

Received 1st May 2017
Accepted 8th June 2017

DOI: $10.1039 / c 7 r a 04885 h$

rsc.li/rsc-advances

electronic structure, charge transport, and surface activity of $\mathrm{TiO}_{2}{ }^{19-28}$ Chen et al. ${ }^{25}$ reported black $\mathrm{TiO}_{2}$ nanoparticles with significant lattice disorder and $\mathrm{Ti}^{3+}$ defects, exhibiting excellent solar-driven photocatalytic activities towards hydrogen generation. Grabstanowicz et al. ${ }^{27}$ have demonstrated that $\mathrm{Ti}^{3+}$-doped $\mathrm{TiO}_{2}$, accompanied with the formation of OVs, displays a broadened absorbance in the visible light region. These imperfections in the crystal lattice lead to lattice distortion, which further affect the corresponding photochemical properties..$^{29-31}$ Still, our understanding with regards to (1) how lattice distortions affect the resulting visible light photoreactivity and (2) the formation of defects remains unclear.

Herein, we have successfully introduced defects based on lattice distortion in $\mathrm{TiO}_{2}$ microspheres. It is demonstrated that the enhancement of visible-light-driven photocatalysis is mainly attributed to the existence of $\mathrm{Ti}^{3+}$ defects, which leads to an elevated valence band edge and a narrower band gap, resulting in higher photoreactivity. The band gap of $\mathrm{TiO}_{2}$ with lattice distortions could not be narrowed, failing to improve visible light photocatalytic activity without $\mathrm{Ti}^{3+}$ defects, but the higher photocurrent generation indicates the migration of recombination of electron-holes pairs. Interestingly, latticedistorted $\mathrm{TiO}_{2}$ favours the formation of $\mathrm{Ti}^{3+}$ defects. A combined effect of lattice distortion and defects was found to increase the photocatalytic activity nearly three times, and the photocurrent generation increased from 1.78 to $80 \mu \mathrm{A} \mathrm{cm} \mathrm{cm}^{-2}$. Such progress is significant for the development of highly efficient photocatalysts and devices for solar fuel generation. 


\section{Experimental}

\section{Synthesis of $\mathrm{TiO}_{2}$-based materials}

All chemicals were of analytical grade and used without further purification. In order to introduce lattice distortions to $\mathrm{TiO}_{2}$ microspheres, $0.05 \mathrm{~mol} \mathrm{Ti}\left(\mathrm{SO}_{4}\right)_{2}$ and $0.05 \mathrm{~mol} \mathrm{NaCl}$ were added to $60 \mathrm{~mL}$ of deionized water. After $30 \mathrm{~min}$ of stirring, the transparent solution was transferred into a high-pressure reactor and heated at $120{ }^{\circ} \mathrm{C}$ for $12 \mathrm{~h}$ with a heating rate of $5{ }^{\circ} \mathrm{C} \min ^{-1}$. Then, the resulting mass was cleaned, dried at $100{ }^{\circ} \mathrm{C}$ for $12 \mathrm{~h}$, and calcined at $300{ }^{\circ} \mathrm{C}$ for $5 \mathrm{~h}$, yielding a white powder. To prepare pure $\mathrm{TiO}_{2}$, no $\mathrm{NaCl}$ was added.

In a typical procedure of introducing defects, $10 \mathrm{~mL}$ ethylene glycol (EG) and $0.3 \mathrm{~g}$ of as-prepared $\mathrm{TiO}_{2}$ were added into a highpressure reactor and were heated at $180^{\circ} \mathrm{C}$ for $5 \mathrm{~h}$ with a heating rate of $5{ }^{\circ} \mathrm{C} \mathrm{min}^{-1}$. The upper transparent solution was discarded and the solid mass was washed and dried at $100{ }^{\circ} \mathrm{C}$ for 12 h. The TG test (Fig. S7†) shows that the EG reduction does not leave organic remains in as-prepared samples.

$\mathrm{TiO}_{2}$ with both lattice distortion and defects was prepared by a combination of experimental procedures described above.

\section{Characterization}

X-ray diffraction (XRD) patterns were collected with a PANalytical Empyrean X-ray diffractometer with monochromatic $\mathrm{Cu}$ $\mathrm{K}_{\alpha}$ radiation $(\lambda=1.5418 \AA)$. X-ray photoelectron spectroscopy (XPS) was carried out on a PHI5300 (PerkinElmer) with a monochromatic $\mathrm{Mg} \mathrm{K} \alpha$ source to analyze the surface chemistry. Scanning electron microscopy (SEM) images were carried out on a field emission scanning electron microscope (JEOL JSM-7001F; acceleration voltage $=10 \mathrm{kV}$ ). Transmission electron microscopy (TEM) images were obtained on a Zeiss LIBRA 200 FEG transmission electron microscope operating at $200 \mathrm{kV}$. UV-vis diffuse reflectance spectroscopy (DRS) was performed on a Shimadzu UV-2100 spectrophotometer using $\mathrm{BaSO}_{4}$ as the reference.

\section{Photoelectrochemical characterization}

The photoelectrochemical response was measured using a CHI 660B electrochemical workstation with conventional threeelectrode setup under visible-light illumination. To fabricate the working electrode, $1 \mathrm{mg}$ of as-prepared pure $\mathrm{TiO}_{2}$ and chlorine introduced $\mathrm{TiO}_{2}$ microspheres were coated onto a slice of ITO glass with an area of $1.5 \times 1.5 \mathrm{~cm}^{2}$. A $0.5 \times 0.5 \mathrm{~cm}^{2} \mathrm{Pt}$ plate and a saturated calomel electrode were used as the counter reference electrodes, respectively. A $0.1 \mathrm{M} \mathrm{Na} \mathrm{NO}_{4}$ solution was used as the electrolyte and a $500 \mathrm{~W}$ Xe lamp was utilized as the visible light source.

\section{Photocatalytic assessment}

The photocatalytic activity of the as-prepared $\mathrm{TiO}_{2}$ microspheres was evaluated by photocatalytic degradation of a solution containing $20 \mathrm{mg} \mathrm{L}^{-1}$ rhodamine $\mathrm{B}(\mathrm{RhB})$ or $15 \mathrm{mg} \mathrm{L}^{-1}$ phenol irradiated with visible light (500 W Xe lamp and a UV cutoff filter $(\lambda>420 \mathrm{~nm}))$. In a typical process, $70 \mathrm{mg}$ of the as- prepared sample was added to $100 \mathrm{~mL}$ of $\mathrm{RhB}$ or phenol. Afterwards, the photocatalyst was dispersed in the solution and stirred for $0.5 \mathrm{~h}$ in the dark to reach adsorption equilibrium before being exposed to visible-light irradiation. The suspension was sampled every $20 \mathrm{~min}$, and the photocatalyst was removed by centrifugation. The change in $\mathrm{RhB}$ or phenol concentration was determined relative to the extinction spectra for RhB by UV-vis spectroscopy.

\section{Results and discussion}

XRD patterns (Fig. 1a) show that all samples are composed of the pure anatase phase, which suggests that there was no significant change in the phase structure by the addition of chloride ions during the hydrothermal processing or reduction with ethylene glycol (EG). Nevertheless, it is noted that the (101) and (004) diffraction peaks slightly shift to smaller angles after adding chloride ions, while no obvious shift is observed after EG reduction, implying that the lattice parameters of $\mathrm{TiO}_{2}$ become larger and lattice distortion is introduced by adding chloride ions. It is further confirmed that the introduction of chlorine ions during the synthetic procedures brings about surface stress in the titania crystals, which results in lattice distortion. All samples are mainly composed of microspheres with average diameters of about $350 \mathrm{~nm}$ (Fig. 1b). Our results suggest that reduction with EG has no effect on the morphology, however, the existence of $\mathrm{Cl}$ ions during hydrothermal processing has a strong effect on the morphology of $\mathrm{TiO}_{2}$ (Fig. S1 $\dagger$ ). The presence of chloride ions leads to more hollow structures in the samples, indicating that the existence of chloride ions may play the role as the surface surfactant $t^{4,32,37}$ (Fig. S2†). TEM analysis further demonstrates that the hollow microspheres are constructed of numerous nanostructures (Fig. 1c). The lattice in area I matches the (101) crystallographic plane of anatase with $d$ spacing of $0.36 \mathrm{~nm}$, indicating that the exposed facets on the surface hold no significant changes (Fig. 1c). Nonetheless, evident lattice distortion exists in the $\mathrm{TiO}_{2}$ with the addition of chloride ions, as shown in Fig. 1c and S3, $\dagger$ which is consistent with the XRD results.

XPS analysis was performed to identify the existence of chloride ions and defects on the surface of the different $\mathrm{TiO}_{2}$ samples. In all samples, there was no signal for chlorine (Fig. S4 $\uparrow$ ), suggesting that the existence of chloride ions in the hydrothermal processing only leads to lattice distortion in-stead of doping and can be removed with subsequent sample washing. Fig. 2 shows the Ti 2p peaks, where the 2p spin-orbital doublets two peaks center at $\sim 458.8 \mathrm{eV}$ and $\sim 464.6 \mathrm{eV}$ correspond to the characteristic Ti $2 \mathrm{p}_{3 / 2}$ and Ti $2 \mathrm{p}_{1 / 2}$ peaks of $\mathrm{Ti}^{4+}$, respectively. ${ }^{33,34} \mathrm{~A}$ detectable shoulder was observed at lower binding energies for the samples that contain defects, which can be ascribed to $\mathrm{Ti}^{3+}$ ions. ${ }^{35,36}$ More importantly, the peak area ratio of $\mathrm{Ti}^{3+} / \mathrm{Ti}^{4+}$ increases from $0.256: 1$ to $0.488: 1$ when lattice distortion is also present in $\mathrm{TiO}_{2}$. This increase in the $\mathrm{Ti}^{3+}$ concentration can be ascribed to the presence of lattice distortion, which allows for more accessible sites for $\mathrm{Ti}^{4+}$ to be reduced to $\mathrm{Ti}^{3+}$. 

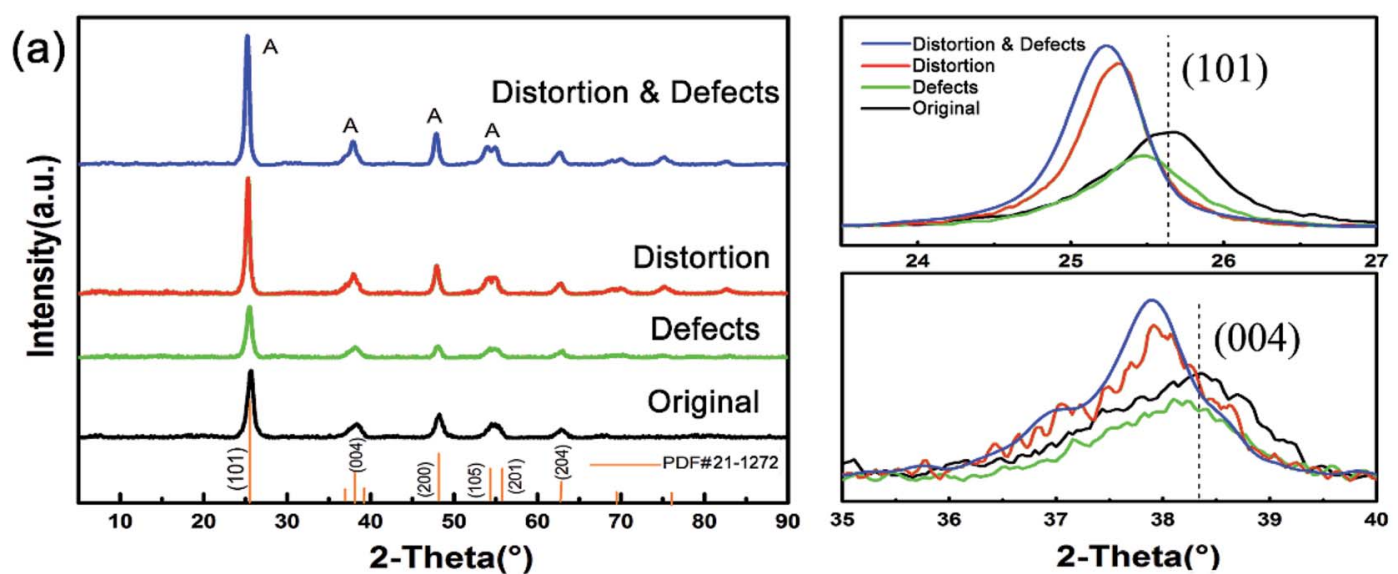

(b)
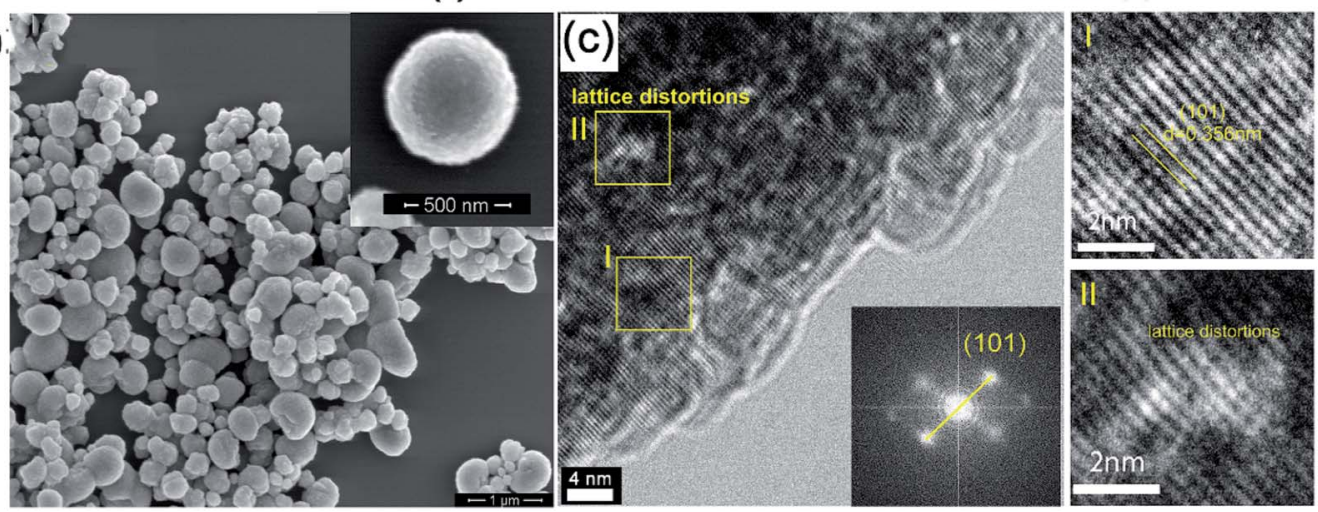

Fig. 1 (a) XRD patterns of the as-prepared $\mathrm{TiO}_{2}$ samples (pristine $\mathrm{TiO}_{2}$ (Original), pure $\mathrm{TiO}_{2}$ with lattice with $\mathrm{Ti}^{3+}$ defects (Defects), pure TiO 2 with lattice distortion (Distortion), $\mathrm{TiO}_{2}$ with both lattice distortion and defects (Distortion \& Defects)). Patterns specially displaying the (101) and (004) peaks are shown to the right. (b) SEM images of the as-prepared $\mathrm{TiO}_{2}$ microspheres with both lattice distortion and defects. (c) HRTEM image of $\mathrm{TiO}_{2}$ with lattice distortion (inset: Fast Fourier Transformation (FFT) for area I) with enlarged HRTEM images of selected areas (I) and (II).

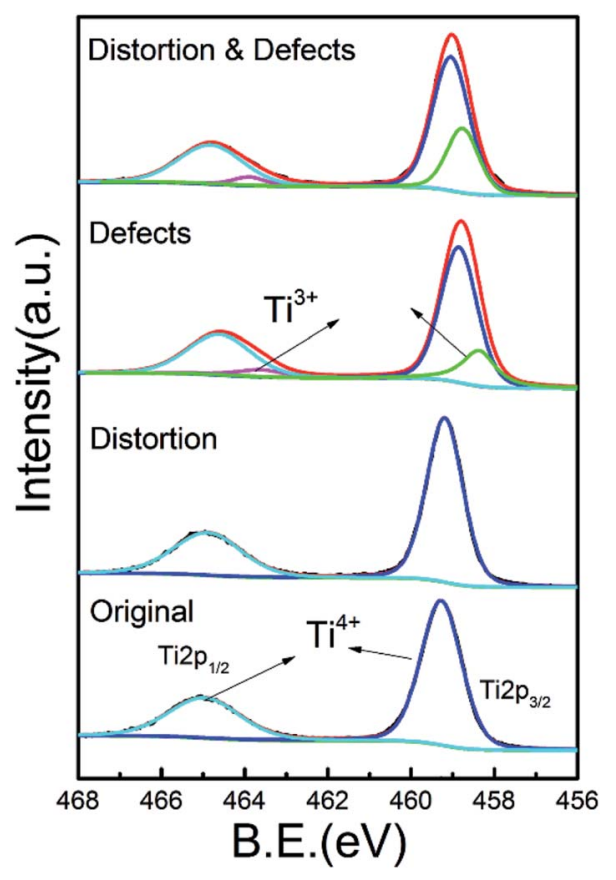

Fig. 2 Ti 2p XPS spectrum of as-prepared $\mathrm{TiO}_{2}$.
The photocatalytic activity of the different $\mathrm{TiO}_{2}$ samples was evaluated by the degradation of rhodamine $\mathrm{B}(\mathrm{RhB})$ dye as well as phenol (Fig. S8†) under visible light irradiation $(\lambda>$ $420 \mathrm{~nm}$ ) (Fig. 3a). Only $37.8 \% \mathrm{RhB}$ is degraded after $120 \mathrm{~min}$ by the original $\mathrm{TiO}_{2}$. When lattice distortion is present in the $\mathrm{TiO}_{2}$ microspheres, the degradation rate of RhB slightly decreases, indicating that lattice distortion due to the introduction of chloride ions during the preparation of $\mathrm{TiO}_{2}$ microspheres fails to enhance the photocatalytic activity of $\mathrm{TiO}_{2}$. After introducing $\mathrm{Ti}^{3+}$ defects, $47.9 \% \mathrm{RhB}$ is degraded after $120 \mathrm{~min}$ using the same reaction conditions, whereas $\mathrm{TiO}_{2}$ with both distortion and defects achieved a degradation of more than $75 \%$. The degradation rate increases dramatically from $38 \times$ $10^{-4}$ to $95 \times 10^{-4} \mathrm{~s}^{-1}$ from $\mathrm{TiO}_{2}$ with just defects to $\mathrm{TiO}_{2}$ with both distortion and defects, displaying that the activity is nearly three times higher when both lattice distortion and defects exist in $\mathrm{TiO}_{2}$ microspheres (Fig. S5 $\dagger$ ). The transient photocurrent response spectra (Fig. $3 \mathrm{~b}$ ) reveal that lattice distortion elevates the photocurrent from 1.78 to $15.56 \mu \mathrm{A}$ $\mathrm{cm}^{-2}$ in the $\mathrm{TiO}_{2}$ microspheres, while $\mathrm{Ti}^{3+}$ defects elevate the photocurrent density to $53.78 \mu \mathrm{A} \mathrm{cm}^{-2}$. Even though the lattice distortion has no contribution on the improvement of visible light photocatalytic activity, the increase of photocurrent suggests that lattice distortion in $\mathrm{TiO}_{2}$ could efficiently supress 

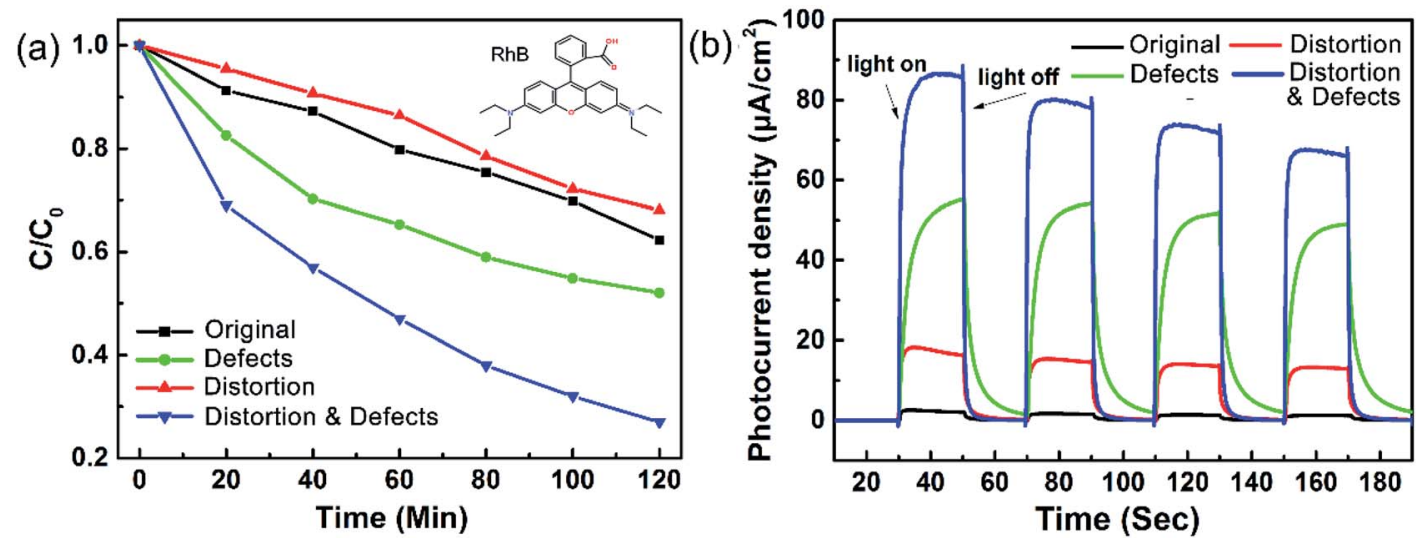

Fig. 3 (a) The degradation of $\mathrm{RhB}$ and (b) photocurrent response of the different $\mathrm{TiO}_{2}$ samples under visible light irradiation.

the recombination of electron-hole pairs. ${ }^{38}$ The existence of both lattice distortion and $\mathrm{Ti}^{3+}$ defects elevates the photocurrent density to $80 \mu \mathrm{A} \mathrm{cm}^{-2}$. The larger the photocurrent value, the higher the charge separation efficiency. Thus, it can be concluded that the introduction of $\mathrm{Ti}^{3+}$ defects facilitates the separation of photoelectrons and holes, which is in accordance with the study of Cai et al. ${ }^{24}$ Considering the effect of surface area, as listed in Table $\mathrm{S} 1, \dagger$ the $\mathrm{TiO}_{2}$ with both defects and lattice distortion possess lower surface areas than the original $\mathrm{TiO}_{2}$, implying that the surface area is not the major effect on the improvement of visible light photoreactivity. It is thereby deduced that the enhancement in the photocurrent generation can be mainly attributed to the existence of $\mathrm{Ti}^{3+}$ defects. The introduction of lattice distortion could not only mitigate the recombination of photo-induced electron-hole pairs, ${ }^{39,45}$ but was also found to be advantageous for the generation of $\mathrm{Ti}^{3+}$ defects. These results demonstrate that the $\mathrm{Ti}^{3+}$ defects play a dominant role in improving the photoreactivity of $\mathrm{TiO}_{2}$ rather than lattice distortion, and a combined effect of lattice distortion and $\mathrm{Ti}^{3+}$ defects is beneficial for the enhancement of visible-light-driven photocatalytic activity and photocurrent generation.

A schematic is presented in Fig. 4 to illustrate the contribution of surficial lattice distortion to the formation of $\mathrm{Ti}^{3+}$ defects. Previous work reported that the effect of adding halogen ions during the sample preparation could tune the

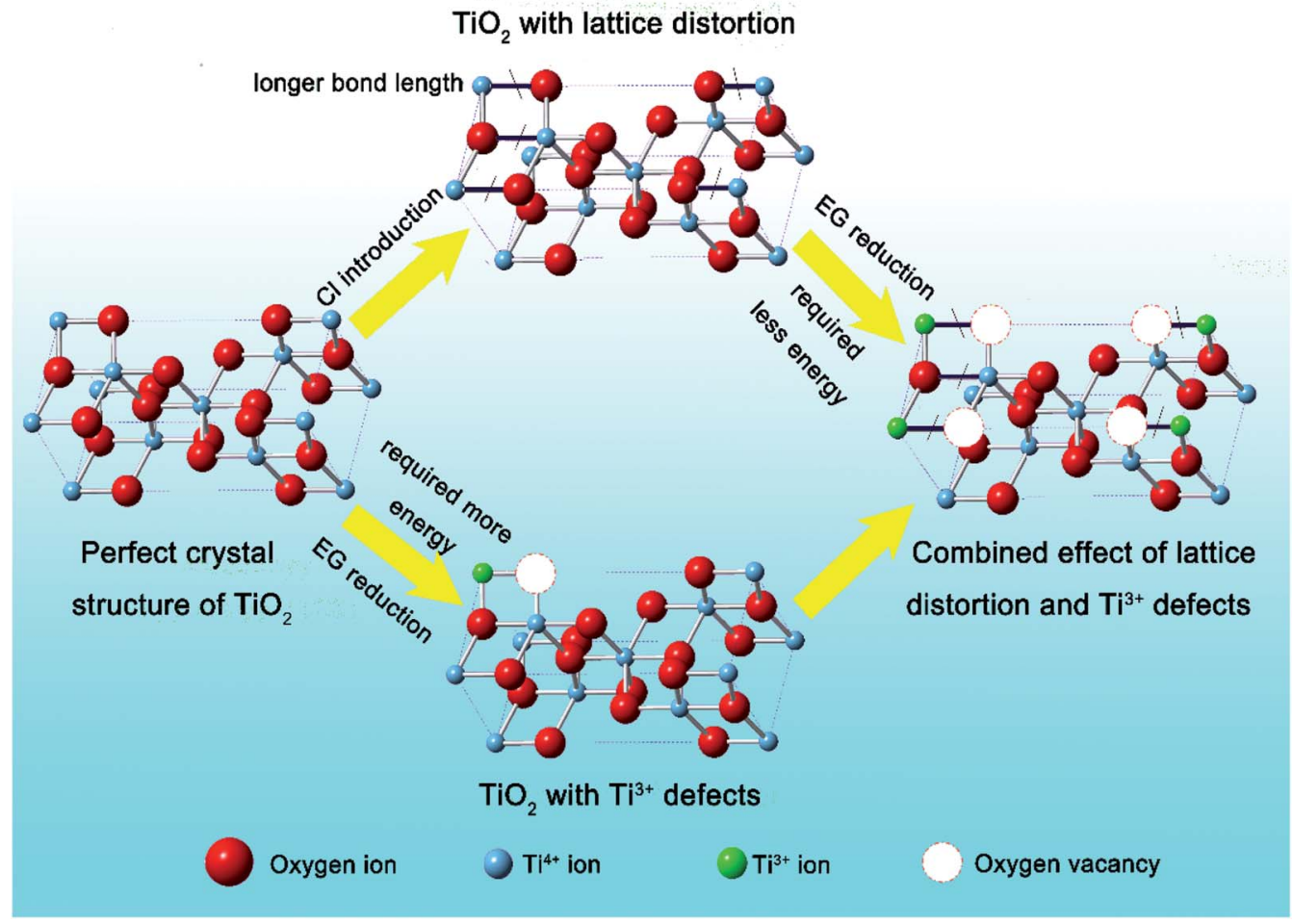

Fig. 4 Schematic illustrating how lattice distortion contributes to the introduction of oxygen vacancies. 
surface morphology due to the higher electronegativity of the halogen atoms, ${ }^{37-39}$ resulting in lattice expansion on the surface of $\mathrm{TiO}_{2}$. This increased bond length would energetically favour the generation of oxygen vacancies from $\mathrm{TiO}_{2}$ due to a lower bonding energy ${ }^{40}$ Then the oxygen deficiencies transfers their extra two electrons to the adjacent two $\mathrm{Ti}^{4+}$ atoms to form $\mathrm{Ti}^{3+}{ }^{31}$ Hence, $\mathrm{Ti}^{3+}$ defects appear and the proportion of these defects is increased for $\mathrm{TiO}_{2}$ with increased amounts of lattice distortion. Finally, the formation of $\mathrm{Ti}^{3+}$ defects for $\mathrm{TiO}_{2}$ with lattice distortion leads to the enhancement of photocurrent generation under visible light while also boosting the photocatalytic activity of $\mathrm{TiO}_{2}$.

Fig. 5a displays the UV-vis diffuse reflectance spectra and the color of the different $\mathrm{TiO}_{2}$ samples. The color changes from white to yellow, and a noticeable difference in the absorption of visible light is observed when $\mathrm{Ti}^{3+}$ defects exist. Valence band (VB) XPS spectra (Fig. 5b) aided in determining the band position for the different $\mathrm{TiO}_{2}$ samples. The pure $\mathrm{TiO}_{2}$ shows a VB maximum energy of $3.10 \mathrm{eV}$ below the Fermi level. After $\mathrm{Ti}^{3+}$ defects were introduced, ${ }^{43-45}$ an obvious upward shift by $0.36 \mathrm{eV}$ occurs in the VB while lattice distortion only brings a slight upward shift by $0.14 \mathrm{eV}$. When both lattice distortion and defects exist, the VB top shifts to $2.64 \mathrm{eV}$ with a $0.46 \mathrm{eV}$ shift from the original $\mathrm{TiO}_{2}$. After introducing defects, a VB tail with a difference of $0.38 \mathrm{eV}$ and $0.46 \mathrm{eV}$ appears in $\mathrm{TiO}_{2}$ with and without lattice distortion, respectively. The corresponding band gaps were calculated ${ }^{42}$ to be $3.06 \mathrm{eV}$ for the $\mathrm{TiO}_{2}$ with lattice distortion, similar to the band gap for the original $\mathrm{TiO}_{2}$ The band gap was found to be $2.98 \mathrm{eV}$ after $\mathrm{Ti}^{3+}$ defects were introduced (Fig. S6 $\dagger$ ). It is thus deduced that the $\mathrm{Ti}^{3+}$ defects (rather than lattice distortion) lead to the extension of the visible light absorption. The VB edge of $\mathrm{TiO}_{2}$ with $\mathrm{Ti}^{3+}$ defects results in a narrower band gap and higher visible light activity when compared to pristine $\mathrm{TiO}_{2}$, which is in line with the results of Pan $e t a l .{ }^{28} \mathrm{~A}$ schematic illustration of the energy band structure for the different $\mathrm{TiO}_{2}$ samples is presented in (Fig. 5c). Due to the contribution of VB tails, the band gap is narrowed to $2.60 \mathrm{eV}$ and further to $2.52 \mathrm{eV}$ as the proportion of $\mathrm{Ti}^{3+}$ defects increases. Therefore, lattice distortion has little influence on the visible light photoreactivity of $\mathrm{TiO}_{2}$, while it benefits the migration and recombination of electron-hole pairs, and both $\mathrm{Ti}^{3+}$ defects and lattice distortion, in turn, greatly improve the visible light photoreactivity of $\mathrm{TiO}_{2}$.
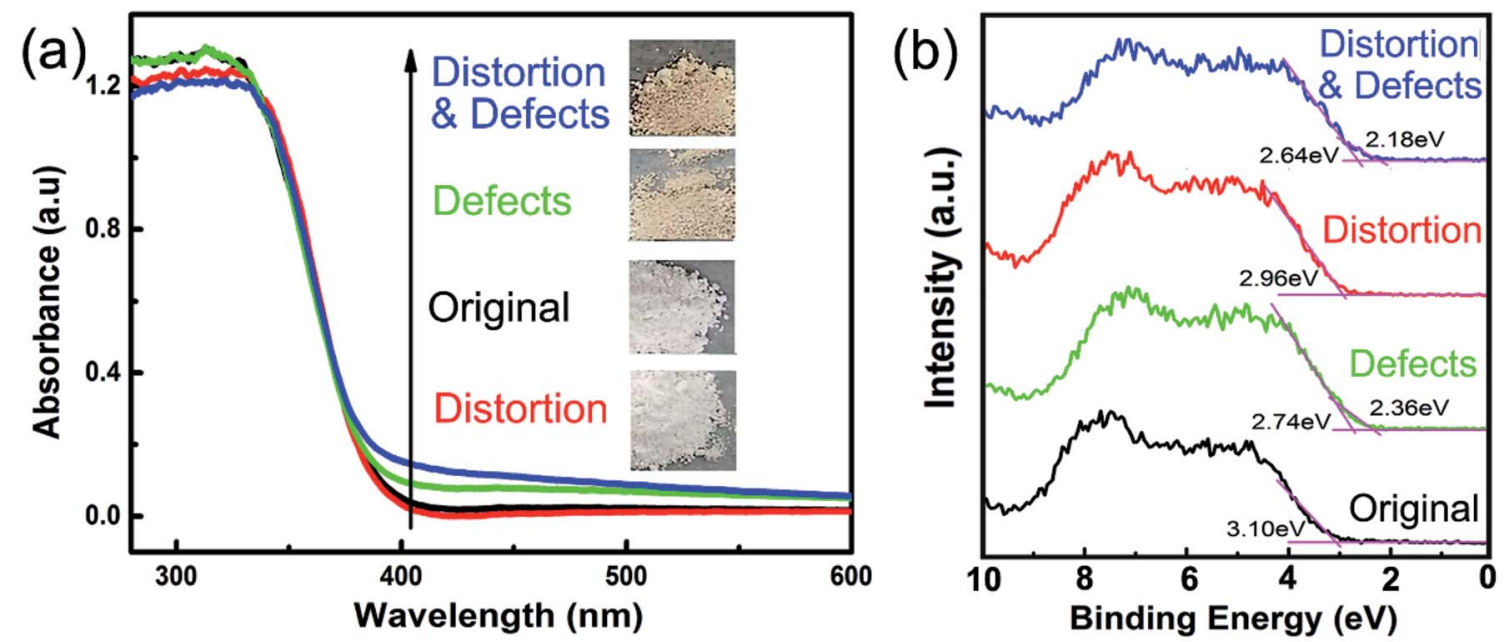

(c)

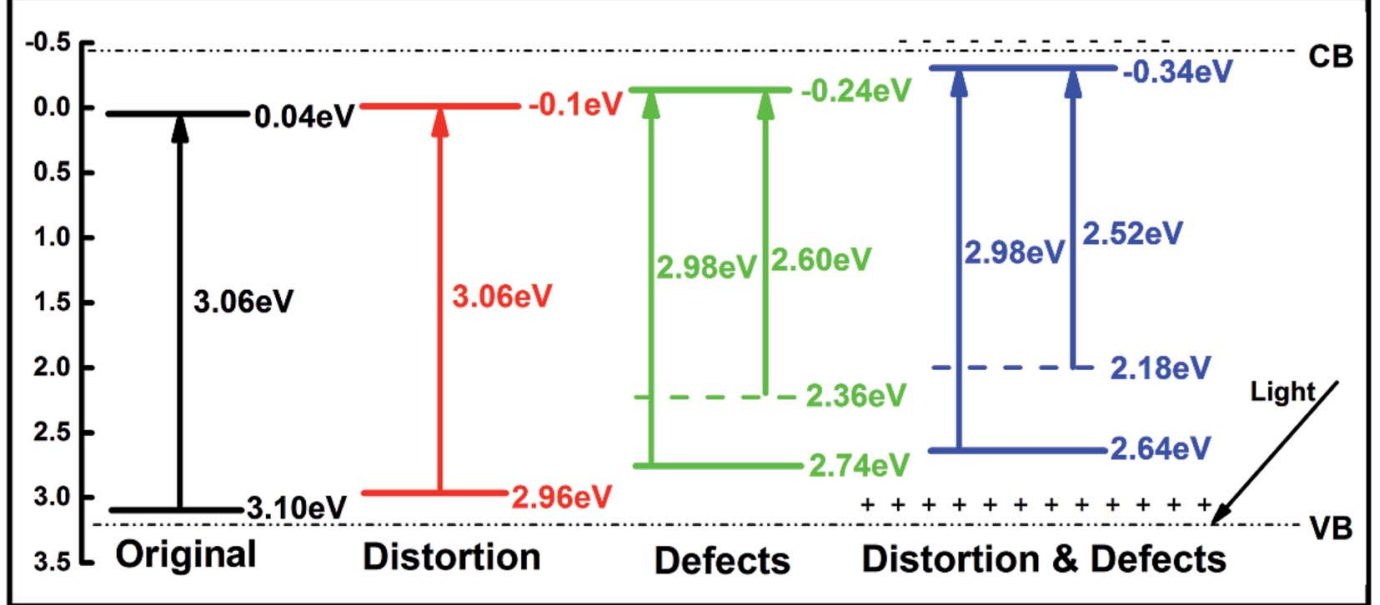

Fig. 5 (a) UV-vis diffuse reflectance spectra. (b) Valence band XPS spectra of the different $\mathrm{TiO}_{2}$ samples. (c) Schematic illustration of the energy band structure for the different $\mathrm{TiO}_{2}$ samples. 


\section{Conclusions}

In conclusion, lattice distortion was introduced to $\mathrm{TiO}_{2}$ microspheres by introducing chlorine during the hydrothermal processing while defects $\left(\mathrm{Ti}^{3+}\right)$ were introduced by reduction with ethylene glycol. Our results demonstrate the relationship between lattice distortion and $\mathrm{Ti}^{3+}$ defects for anatase, which were strongly correlated with their photocatalytic properties. The existence of $\mathrm{Ti}^{3+}$ defects was found to strengthen the absorption of light in the UV and visible light regions and further elevates the VB position, leading to overall improved photocatalytic activity towards the degradation of RhB and phenol under visible light irradiation. Our results also demonstrate that defects and lattice distortion enhance the photocurrent generation of anatase $\mathrm{TiO}_{2}$ under visible light. $\mathrm{Ti}^{3+}$ defects are energetically favored over lattice distortion in $\mathrm{TiO}_{2}$ compared to that in pristine anatase $\mathrm{TiO}_{2}$. This study sheds light on the mechanism for engineering materials with lattice disorder and opens new opportunities for the design and synthesis of high-performance photocatalysts and solar fuel generation devices that operate under visible light irradiation.

\section{Acknowledgements}

This research is financially supported by the Fundamental Research Funds for the Central Universities (No. 106112015CDJXY130010) and the National Natural Science Foundation of China (No. 51302329). We gratefully acknowledge the theoretical calculations processed by Dr Wen Zeng. The preparation of this manuscript was also supported by the Center for Nanophase Materials Sciences, which is a DOE Office of Science User Facility. ZDH gratefully acknowledges a graduate fellowship from the National Science Foundation under Grant No. DGE-1148903 and the Georgia Tech-ORNL Fellowship.

\section{Notes and references}

$1 \mathrm{~J}$. H. Carey, J. Lawrence and H. M. Tosine, Photodechlorination of PCB's in the Presence of Titanium Dioxide in Aqueous Suspensions, Bull. Environ. Contam. Toxicol., 1976, 16, 697-701.

2 X. Pan, M. Q. Yang and X. Fu, Defective $\mathrm{TiO}_{2}$ with Oxygen Vacancies: Synthesis, Properties and Photocatalytic Applications, Nanoscale, 2013, 5, 3601-3614.

3 A. L. Linsebigler, G. Lu and J. T. Yates Jr, Photocatalysis on $\mathrm{TiO}_{2}$ Surfaces: Principles, Mechanisms, and Selected Results, Chem. Rev., 1995, 95, 735-758.

4 M. Kapilashrami, Y. Zhang, Y. S. Liu, et al., Probing the Optical Property and Electronic Structure of $\mathrm{TiO}_{2}$ Nanomaterials for Renewable Energy Applications, Chem. Rev., 2014, 114, 9662-9707.

5 W. J. Ong, L. L. Tan and S. P. Chai, Self-assembly of Nitrogendoped $\mathrm{TiO}_{2}$ with Exposed $\{001\}$ Facets on a Graphene Scaffold as Photo-active Hybrid Nanostructures for Reduction of Carbon Dioxide to Methane, Nano Res., 2014, 7, 1528-1547.
6 J. C. Wu, D. S. Liu and A. N. Ko, Dehydrogenation of Ethylbenzene over $\mathrm{TiO}_{2}-\mathrm{Fe}_{2} \mathrm{O}_{3}$ and $\mathrm{ZrO}_{2}-\mathrm{Fe}_{2} \mathrm{O}_{3}$ Mixed Oxide Catalysts, Catal. Lett., 1993, 20, 191-201.

7 K. Vinodgopal, I. Bedja and P. V. Kamat, Nanostructured Semiconductor Films for Photocatalysis. Photoelectrochemical Behavior of $\mathrm{SnO}_{2} / \mathrm{TiO}_{2}$ Composite Systems and its Role in Photocatalytic Degradation of a Textile Azo Dye, Chem. Mater., 1996, 8, 2180-2187.

8 R. Sasikala, A. Shirole, V. Sudarsan, et al., Highly Dispersed Phase of $\mathrm{SnO}_{2}$ on $\mathrm{TiO}_{2}$ Nanoparticles Synthesized by Polyol-mediated Route: Photocatalytic Activity for Hydrogen Generation, Int. J. Hydrogen Energy, 2009, 34, 3621-3630.

9 C. Yu, G. Li and S. Kumar, Stable Au25 (SR) 18/ $\mathrm{TiO}_{2}$ Composite Nanostructure with Enhanced Visible Light Photocatalytic Activity, J. Phys. Chem. Lett., 2013, 4, 28472852.

10 H. Sun, S. Wang, H. M. Ang, et al., Halogen Element Modified Titanium Dioxide for Visible Light Photocatalysis., Chem. Eng. J., 2010, 162, 437-447.

11 X. Liu, P. Lv and G. Yao, Effection of Chlorine-doped $\mathrm{TiO}_{2}$ Photocatalysts for Photocatalytic Activity, Asian J. Chem., 2013, 25, 3275.

12 R. Yuan, T. Chen and E. Fei, Surface Chlorination of $\mathrm{TiO}_{2}$ based Photocatalysts: A Way to Remarkably Improve Photocatalytic Activity in Both UV and Visible Region, ACS Catal., 2011, 1, 200-206.

13 S. Horikoshi, T. Miura and M. A. Kajitani, FT-IR (DRIFT) Study of the Influence of Halogen Substituents on the $\mathrm{TiO}_{2}$-assisted Photooxidation of Phenol and $p$-halophenols under Weak Room Light Irradiance, J. Photochem. Photobiol., A, 2008, 194, 189-199.

14 W. Choi, A. Termin and M. R. Hoffmann, The Role of Metal Ion Dopants in Quantum-sized $\mathrm{TiO}_{2}$ : Correlation between Photoreactivity and Charge Carrier Recombination Dynamics, J. Phys. Chem., 1994, 98, 13669-13679.

15 I. Y. Jeon, H. J. Choi and M. Choi, Facile, Scalable Synthesis of Edge-halogenated Graphene Nanoplatelets as Efficient Metal-free Electrocatalysts for Oxygen Reduction Reaction, Sci. Rep., 2013, 3, 1810.

16 J. Wang and Z. Lin, Dye-sensitized $\mathrm{TiO}_{2}$ Nanotube Solar Cells with Markedly Enhanced Performance via Rational Surface Engineering, Chem. Mater., 2009, 22, 579-584.

17 N. Guijarro, T. Lana-Villarreal, T. Lutz, et al., Sensitization of $\mathrm{TiO}_{2}$ with PbSe Quantum Dots by SILAR: How Mercaptophenol Improves Charge separation, J. Phys. Chem. Lett., 2012, 3, 3367-3372.

18 W. T. Sun, Y. Yu, H. Y. Pan, et al., CdS Quantum Dots Sensitized $\mathrm{TiO}_{2}$ Nanotube-array Photoelectrodes, J. Am. Chem. Soc., 2008, 130, 1124-1125.

19 F. Zuo, L. Wang and T. Wu, Self-doped $\mathrm{Ti}^{3+}$ Enhanced Photocatalyst for Hydrogen Production under Visible Light, J. Am. Chem. Soc., 2010, 132, 11856-11857.

20 J. Huo, Y. Hu and H. Jiang, In Situ Surface Hydrogenation Synthesis of $\mathrm{Ti}^{3+}$ Self-doped $\mathrm{TiO}_{2}$ with Enhanced Visible Light Photoactivity, Nanoscale, 2014, 6, 9078-9084.

21 S. Wang, L. Pan, J. J. Song, et al., Titanium-defected undoped anatase $\mathrm{TiO}_{2}$ with p-type conductivity, room-temperature 
ferromagnetism, and remarkable photocatalytic performance, J. Am. Chem. Soc., 2015, 137, 2975-2983.

22 Y. H. Hu, A Highly Efficient Photocatalyst-Hydrogenated Black $\mathrm{TiO}_{2}$ for the Photocatalytic Splitting of Water, Angew. Chem., Int. Ed., 2012, 51, 12410-12412.

23 L. Pan, S. Wang, J. Xie, et al., Constructing $\mathrm{TiO}_{2}$ pn homojunction for photoelectrochemical and photocatalytic hydrogen generation, Nano Energy, 2016, 28, 296-303.

24 J. Cai, K. Lv, J. Sun, et al., Ti powder-assisted synthesis of $\mathrm{Ti}^{3+}$ self-doped $\mathrm{TiO}_{2}$ nanosheets with enhanced visible-light photoactivity, RSC Adv., 2014, 4, 19588-19593.

25 X. Chen, L. Liu and F. Huang, Black Titanium Dioxide $\left(\mathrm{TiO}_{2}\right)$ Nanomaterials, Chem. Soc. Rev., 2015, 44, 1861-1885.

26 A. L. Linsebigler, G. Lu and J. T. Yates Jr, Photocatalysis on $\mathrm{TiO}_{2}$ Surfaces: Principles, Mechanisms, and Selected Results, Chem. Rev., 1995, 95, 735-758.

27 L. R. Grabstanowicz, S. Gao, T. Li, et al., Facile oxidative conversion of $\mathrm{TiH}_{2}$ to high-concentration $\mathrm{Ti}^{3+}$-self-doped rutile $\mathrm{TiO}_{2}$ with visible-light photoactivity, Inorg. Chem., 2013, 52, 3884-3890.

28 L. Pan, S. Wang, J. J. Zou, et al., $\mathrm{Ti}^{3+}$-defected and V-doped $\mathrm{TiO}_{2}$ quantum dots loaded on MCM-41, Chem. Commun., 2014, 50, 988-990.

29 D. Kim, J.-h. Yang and J. Hong, Ferromagnetism induced by Zn vacancy defect and lattice distortion in ZnO, J. Appl. Phys., 2009, 106, 013908.

30 I. M. Lifshitz and A. M. Kosevich, The dynamics of a crystal lattice with defects, Rep. Prog. Phys., 1966, 29(1), 217-254.

31 M. Trau, N. Yao, E. Kim, et al., Microscopic patterning of orientated mesoscopic silica through guided growth, Nature, 1997, 390, 674-676.

32 E. Andrews and S. M. Larson, Effect of surfactant layers on the size changes of aerosol particles as a function of relative humidity, Environ. Sci. Technol., 1993, 27, 857-865.

33 X. Chen, L. Liu and Y. Y. Peter, Increasing Solar Absorption for Photocatalysis with Black Hydrogenated Titanium Dioxide Nanocrystals, Science, 2011, 331, 746-750.

34 M. S. Lazarus and T. K. Sham, X-ray Photoelectron Spectroscopy (XPS) Studies of Hydrogen Reduced Rutile $\left(\mathrm{TiO}_{2-x}\right)$ Surfaces, Chem. Phys. Lett., 1982, 92, 670-674.
35 G. Liu, H. G. Yang and X. Wang, Enhanced Photoactivity of Oxygen-deficient Anatase $\mathrm{TiO}_{2}$ Sheets with Dominant $\{001\}$ Facets, J. Phys. Chem. C, 2009, 113, 21784-21788.

36 C. Di Valentin, G. Pacchioni and A. Selloni, Reduced and ntype doped $\mathrm{TiO}_{2}$ : nature of $\mathrm{Ti}^{3+}$ species, J. Phys. Chem. C, 2009, 113, 20543-20552.

37 T. R. Gordon, M. Cargnello and T. Paik, Nonaqueous Synthesis of $\mathrm{TiO}_{2}$ Nanocrystals Using $\mathrm{TiF}_{4}$ to Engineer Morphology, Oxygen Vacancy Concentration, and Photocatalytic Activity, J. Am. Chem. Soc., 2012, 134, 67516761.

38 H. Tan, A. Jain, O. Voznyy, et al., Efficient and stable solution-processed planar perovskite solar cells via contact passivation, Science, 2017, 355, 722-726.

39 M. Lapertot, P. Pichat and S. Parra, Photocatalytic Degradation of p-halophenols in $\mathrm{TiO}_{2}$ Aqueous Suspensions: Halogen Effect on Removal Rate, Aromatic Intermediates and Toxicity Variations, J. Environ. Sci. Health, Part A: Environ. Sci. Eng., 2006, 41, 1009-1025.

40 L. Ye, L. Zan, L. Tian, et al., The $\{001\}$ facets-dependent high photoactivity of BiOCl nanosheets, Chem. Commun., 2011, 47, 6951-6953.

41 M. Batzill, E. H. Morales and U. Diebold, Surface studies of nitrogen implanted $\mathrm{TiO}_{2}$, Chem. Phys., 2007, 339, 36-43.

42 M. A. Butler, Photoelectrolysis and physical properties of the semiconducting electrode $\mathrm{WO}_{2}$, J. Appl. Phys., 1977, 48, 1914.

43 S. Wang, L. Zhao and L. Bai, Enhancing photocatalytic activity of disorder-engineered $\quad \mathrm{C}^{-\mathrm{TiO}_{2}}$ and $\mathrm{TiO}_{2}$ nanoparticles, J. Mater. Chem. A, 2014, 2, 7439-7445.

44 A. Sinhamahapatra, J. P. Jeon and J. S. Yu, A New Approach to Prepare Highly Active and Stable Black Titania for Visible Light-assisted Hydrogen Production, Energy Environ. Sci., 2015, 8, 3539-3544.

45 A. Naldoni, M. Allieta and S. Santangelo, Effect of Nature and Location of Defects on Bandgap Narrowing in Black $\mathrm{TiO}_{2}$ Nanoparticles, J. Am. Chem. Soc., 2012, 134, 76007603. 